\title{
Bragg grating based integrated photonic Hilbert transformers
}

\author{
Chaotan Sima*, J. C. Gates, M. N. Zervas, and P. G.R. Smith \\ Optoelectronics Research Centre, University of Southampton, Southampton SO17 1BJ, United Kingdom \\ Chaotan.sima@soton.ac.uk
}

\begin{abstract}
Planar Bragg grating based photonic Hilbert transformers are experimentally demonstrated in this work. Planar Bragg gratings are utilized to implement a general Hilbert transform, a fractional order Hilbert transform and terahertz bandwidth Hilbert transforms, using a direct UV grating writing technique in a silica-on-silicon platform. The design, fabrication and integration of the proposed devices are discussed and presented. The photonic Hilbert transformers could be further monolithically integrated with flat top reflectors and interferometric structures, implementing all-optical singlesideband filters.
\end{abstract}

Keywords-Bragg reflectors; gratings; direct $U V$ writing; alloptical signal processing; Hilbert transform

\section{INTRODUCTION}

Hilbert transform (HT) techniques have been widely used in single-sideband (SSB) modulation applications for improving performance in terms of required power, enhancing spectral efficiency and reducing fading, and devices have been implemented in both analogue and digital systems and geometries [1]. In the all-optical field, photonic Hilbert transformers (PHTs) provide wide bandwidth and high speed for applications including characterization of broadband microwave signals [2] and all-optical SSB modulation [3]. In addition they also reduce dispersion related artifacts such as signal power fading [4]. PHTs have been practically realized via various methods, e.g. using fiber Bragg gratings (FBGs) [57], optical ring resonators [8] and planar Bragg gratings [9].

Fractional Hilbert transformers (FrHT) offer a new degree of freedom, the fractional order, which can be used for a better characterization of a signal under test, or as an additional encoding parameter $[10,11]$ with an experimental demonstration presented in this work.

While previously reported experimental approaches have been limited to operation bandwidths $<100 \mathrm{GHz}$, due to intrinsic complexity of their approaches via the use of discrete components or the restrictions of existing FBG fabrication [12, 13], terahertz bandwidth PHTs were numerically presented using long period gratings and core/cladding mode interaction [12], nevertheless, the complexity provides a significant challenge to practically realizing such a device.

In this work, we experimentally demonstrate a direct and simple route to realize Bragg grating based PHTs, with fractional transform order, and even prospects for operation with Terahertz bandwidths. We use the phase-modulated direct
UV grating writing technique in a silica-on-silicon planar format [14], which provides a route to realize compact optical modules for integrated optical applications. These devices have the benefits of compact structures with fiber compatibility, long term thermal stability and could be used alongside etched silica-on-silicon devices..

\section{OPERATION PRINCIPLE}

The conventional Hilbert transform $\mathrm{H}[\mathrm{g}(\mathrm{t})]$ of a onedimensional real signal $\mathrm{g}(\mathrm{t})$ is [1]:

$$
H[g(t)]=\hat{g}(t)=g(t) * \frac{1}{\pi t}=\frac{1}{\pi} P \int_{-\infty}^{\infty} \frac{g(\tau)}{t-\tau} d \tau
$$

where $\mathrm{P}$ stands for the Cauchy principal value [1]. The symbol $*$ denotes the convolution of $g(t)$ and $1 / \pi \mathrm{t}$. The Fourier transform of the kernel of the Hilbert transform is:

$$
H(\omega)=-j \operatorname{sgn}(\omega)
$$

where $\omega$ is the angular frequency and where $\operatorname{sgn}(\omega)$ is the sign function (which is +1 for $\omega>0,0$ for $\omega=0$ and otherwise).

In any practical implementation of Hilbert transform, the output signal is a delayed and somewhat distorted HT of the input signal. As a consequence the frequency response of the

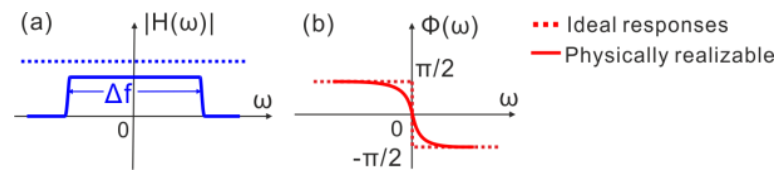

Fig. 1. Schematic of the (a) amplitude and (b) phase responses of a physically realizable HT (solid lines) compared to those of the ideal HT (dashed lines) in the frequency domain.
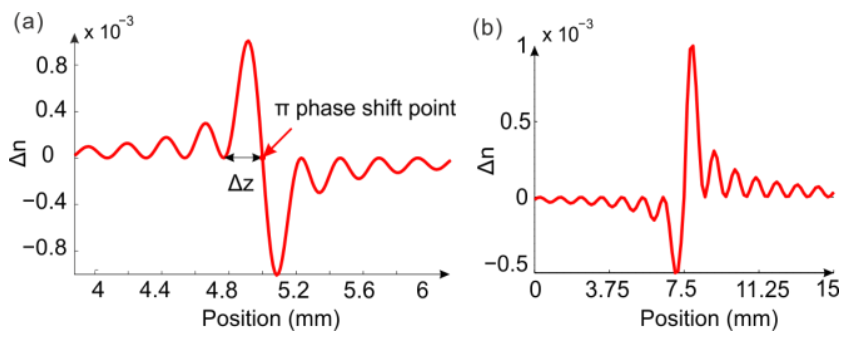

Fig. 2. (a) The central apodization profile of the Hilbert transform grating with $1 \mathrm{THz}$ bandwidth; (b) the apodization profile for fractional order Hilbert transformer. 
HT will be band-limited with a bandwidth $\Delta \mathrm{f}$ and a smooth alteration in phase as illustrated in Fig. 1. The PHT has a $\pi$ phase shift at $\omega=0$, whereas the amplitude remains constant. For a physically realizable PHT, the practical grating apodization profile has been given in [6]:

$$
\Delta n(z) \propto \frac{\sin ^{2}\left[\pi n_{e f f} \Delta f\left(z-z_{0}\right) / c\right]}{z-z_{0}}
$$

where $n_{\text {eff }}$ is the grating effective refractive index, $c$ is the light speed in vacuum, $\mathrm{z}$ is the grating length, $\mathrm{z}_{0}$ is the zerocrossing point in the apodization function, and $\Delta \mathrm{f}$ is the operating bandwidth. From (3), the full operation spectral bandwidth of the PHT is in inverse proportion to the period of the apodization profile $\Delta z$, also refers to the zero-to-zero width of the sidelobe in the apodization profile [6], as shown in Fig. 2(a), given as:

$$
\Delta f=\frac{c}{n_{e f f} \Delta z}
$$

\section{A. Fractional Hilbert transform}

The definition of FrHT was discussed in [10, 11]. A devices implementing FrHT would have a fractional order of $\pi$ phase shift in the frequency response while the amplitude response remains constant. It was reported this phase shift could be continuously adjusted up to a maximum value of $\pi$, by varying the relative maximum index modulation depths $(\Delta \mathrm{n})$ at both sides of the zero-crossing point in the apodization profile, without affecting the flatness in the amplitude response [11], as shown in Fig. 2(b). This behavior allows the direct design and fabrication of an FrHT by using planar Bragg grating structures. A transfer matrix method was employed for modeling the device output with the proposed structure; the modeled data are shown in Fig. 3.
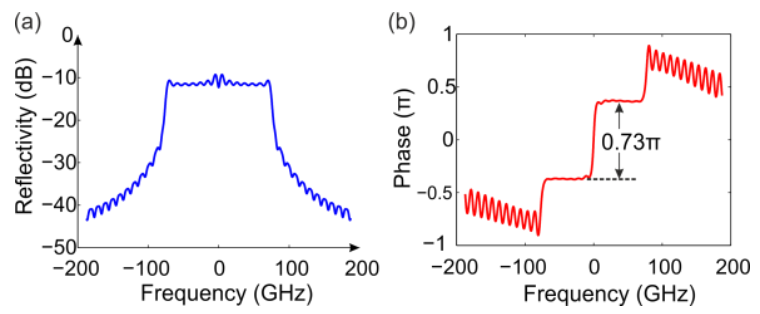

Fig. 3. (a) The modeled grating amplitude response for FrHT ; (b) the modeled grating phase response, showing a fractional order of 0.73 .

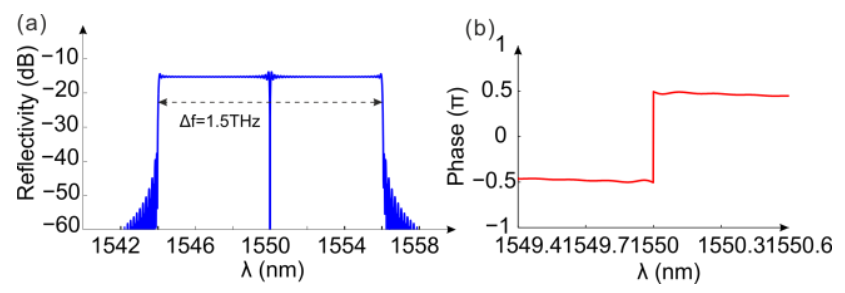

Fig. 4. (a) The modeled reflectivity spectrum of the Hilbert transform grating with $1.5 \mathrm{THz}$ bandwidth; (b) the modeled phase response.
Fig. 3 illustrates (a) the grating amplitude response for FrHT, and (b) the grating phase response, respectively. The average group delay is not represented for illustration purpose. The Bragg grating is set to $15 \mathrm{~mm}, \mathrm{n}_{\text {eff }}=1.4478$, and $\Delta \mathrm{f}=$ $150 \mathrm{GHz}$. By reducing the maximum index modulation depth $(\Delta \mathrm{n})$ on one side of the zero-crossing point in the apodization profile in Fig. 2(b), a fractional order of $\pi$ phase shift was achieved, i.e. 0.73 in Fig. 3(b), which is an essential feature of fractional Hilbert transform.

\section{B. Terahertz bandwidth Hilbert transform}

Terahertz bandwidth Hilbert transform have the benefit of implementing PHT devices for processing ultra wideband signals with terahertz bandwidth. A broader processing bandwidth can be obtained using narrower apodization sidelobe in (4). Assuming the grating length is $10 \mathrm{~mm}, \mathrm{n}_{\mathrm{eff}}=$ 1.45 and the maximum refractive index modulation depth was $\Delta \mathrm{n}=9 \times 10^{-4}$. Fig. 4 illustrates the reflectivity and phase spectra of the proposed Bragg gratings, with $1.5 \mathrm{THz}$ bandwidth.

\section{EXPERIMENTAL DEMONSTRATION}

The proposed planar structures were fabricated using the direct UV grating writing technique $[14,15]$, shown in Fig. 5. This method involves focusing two crossed laser beams $(\lambda=$ $244 \mathrm{~nm}$ ) into the photosensitive core of a planar sample. Precise translation of the sample and modulation of the interference pattern creates grating structures and simultaneously defines the channel waveguide. This spot size is $\sim 6 \mu \mathrm{m}$ in diameter, providing the unique ability over traditional FBG to manipulate the grating's structure at the micron level.

The fabricated devices had a three-layer silica-on-silicon

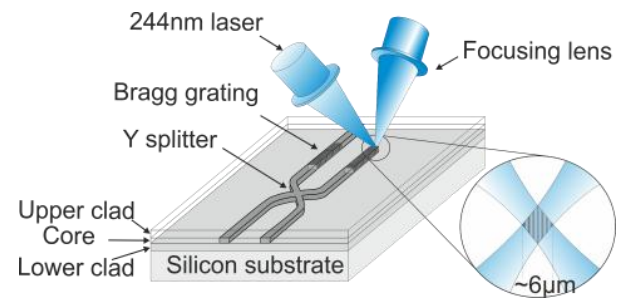

Fig. 5. Direct Grating writing demo, showing crossed beams focused on the core layer of the silica-on-silicon substrate, with the writing spot in $\sim 6$ $\mu \mathrm{m}$ diameter.

substrate, containing a thermal oxide layer (lower clad), core and an upper clad. The Bragg gratings sections were all $10 \mathrm{~mm}$ long, the effective refractive index of the mode within the gratings was 1.447. Fig. 6 shows the modeled data (blue line) and the measured data (red dashed line) of the grating amplitude response for (a) the 0.4 order FrHT and (b) the 0.5 order FrHT. Data in Fig. 6 were measured using an optical spectrum analyzer (OSA), 3dB coupler and an erbium doped fiber amplified spontaneous emission (ASE) source. The measurement system has a noise floor of about $-30 \mathrm{~dB}$.

Terahertz bandwidth PHTs were also fabricated using the same technique. Fig. 7 shows the measured grating (a) reflectivity and (b) relative group delay responses, of the PHT with $1.5 \mathrm{THz}$ bandwidth. Due to lack of availability of a direct 


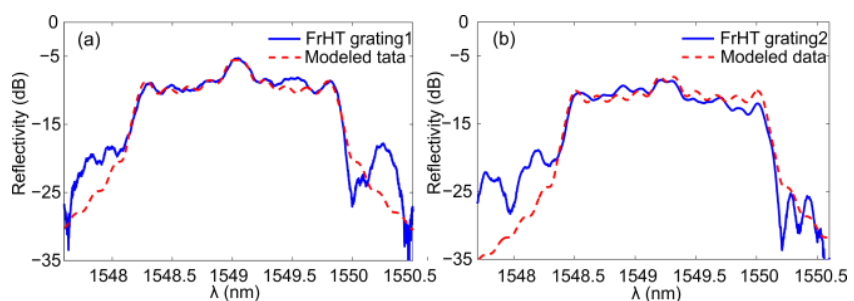

Fig. 6. Measured grating reflectivity spectra (blue line), with modeled data (red dashed line): (a) 0.4 order FrHT and (b) 0.5 order FrHT.
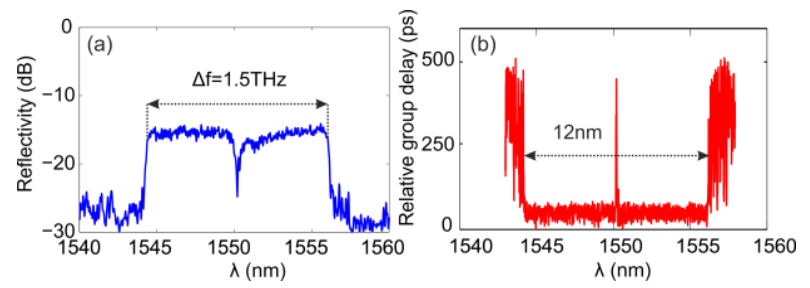

Fig. 7.(a) The measured reflectivity spectrum of the fabricated Bragg gratings implementing $1.5 \mathrm{THz}$ bandwidth PHT; (b) the measured relative group delay of the planar Bragg grating.

optical phase measurement system, a modulation phase-shift method [16] was used to obtain relative group delay data instead. In Fig. 7(a), the PHT shows a flat-top amplitude response, with $\sim 2 \mathrm{~dB}$ ripples for $1.5 \mathrm{THz}$ bandwidth. Fig. 7(b) indicates the $\sim \pi$ phase shift at the central dip of the amplitude spectra. We can use the active phase control, e.g. thermal tuning [14], to compensate any PHT phase error. This initial proof-of-concept device demonstrates $12 \mathrm{~nm} \quad(\sim 1.5 \mathrm{THz})$ operation bandwidth PHT.

\section{CONCLUSTION}

In summary, we have experimentally demonstrated a series of photonic Hilbert transformers based on planar Bragg gratings, featuring operation with fractional order and featuring terahertz bandwidths. PHT devices displaying the 0.5 fractional order and the $1.5 \mathrm{THz}$ operation bandwidth have been fabricated via the direct UV grating writing technique. This fabrication technique allows precise engineering of the amplitude and phase response of the gratings with complex structures at micron level. The PHTs can be further integrated with couplers and flat-top reflectors to perform ultra wideband all-optical SSB filters.

\section{ACKNOWLEDGMENT}

This work was supported by Engineering and Physical Sciences Research Council (EPSRC), UK and China Scholarship Council, China.

\section{REFERENCES}

[1] S. L. Hahn, in The Transforms and Applications Handbook, 2nd ed., A.D. Poularikas, ed. Boca Raton: CRC Press, 2000.

[2] H. Emami, N. Sarkhosh, L. A. Bui, and A. Mitchell, "Amplitude independent RF instantaneous frequency measurement system using photonic Hilbert transform," Opt. Express, vol. 16, pp. 13707-13712, 2008.

[3] K. Takano, N. Hanzawa, S. Tanji and K. Nakagawa, "Experimental demonstration of optically phase-shiftd SSB modulation with Fiberbased optical Hilbert transformers," in Optical Fiber Communication and the National Fiber Optic Engineers Conference, Technical Digest (Optical Society of America, Anaheim, CA, 2007), Paper JThA48.

[4] Z. Li, H. Chi, X. Zhang, and J. P. Yao, "Optical single-sideband modulation using a fiber-Bragg-grating-based optical Hilbert transformer," IEEE Photon. Technol. Lett., vol. 23, pp. 558-560, 2011.

[5] X. Wang, M. Hanawa, K. Nakamura, K. Takano and K. Nakagawa, "Sideband Suppression Characteristics of Optical SSB Generation Filter with Sampled FBG Based 4-taps Optical Hilbert Transformer," in Proceedings of the 15th Asia-Pacific Conference on Communications (APCC, 2009), pp. 622-625, 2009.

[6] M. H. Asghari and J. Azaña, "All-optical Hilbert transformer based on a single phase-shifted fiber Bragg grating: Design and analysis," Opt. Lett.,vol. 34, pp. 334-336, 2009.

[7] M. Li and J. Yao, "Experimental Demonstration of a Wideband Photonic Temporal Hilbert Transformer Based on a Single Fiber Bragg Grating,” IEEE Photon. Technol. Lett., vol. 22, pp. 1559-1561, 2010.

[8] L. Zhuang, M. R. Khan, W. Beeker, A. Leinse, R. Heideman, and C. Roeloffzen, "Novel microwave photonic fractional Hilbert transformer using a ring resonator-based optical all-pass filter," Opt. Express, vol. 20, pp. 26499-26510, 2012.

[9] C. Sima, J. C. Gates, H. L. Rogers, P. L. Mennea, C. Holmes, M. N. Zervas, and P. G. R. Smith, "Phase controlled integrated interferometric single-sideband filter based on planar Bragg gratings implementing photonic Hilbert transform," Opt. Lett., vol. 38, pp. 727-729, 2013.

[10] A. W. Lohmann, D. Mendlovic, and Z. Zalevsky, "Fractional hilbert transform," Opt. lett., vol. 21, no. 4, pp. 281-283, 1996

[11] C. Cuadrado-Laborde, "Proposal and design of a photonic in-fiber fractionalhilbert transformer," Photonics Technology Letters, IEEE, vol. 22, pp. 33-35, 2010.

[12] R. Ashrafi, and J. Azaña, "Terahertz bandwidth all-optical Hilbert transformers based on long-period gratings," Optics Letters, vol. 37, pp. 2604-2606, 2012.

[13] C. Sima, J. C. Gates, M. N. Zervas, and P. G.R. Smith, "Review of photonic Hilbert transformers," Frontiers of Optoelectronics, vol. 6, pp. 78-88, 2013.

[14] C. Sima, J. C. Gates, P. L. Mennea, C. Holmes, M. N. Zervas, and P. G. R. Smith, "Ultra-wide detuning planar Bragg grating fabrication technique based on direct UV grating writing with electro-optic phase modulation", Opt. Express, under peer review.

[15] G. Emmerson, S. Watts, C. Gawith, V. Albanis, M. Ibsen, R. Williams, and P. Smith, "Fabrication of directly uv-written channel waveguides with simultaneously defined integral bragg gratings," Electron. Lett., vol. 38, pp. 1531-1532, 2002.

[16] P. A. Williams, "Modulation phase-shift measurement of PMD using only four launched polarisation states: a new algorithm," Electron. Lett., vol. 35, pp. 1758-1579, 1999. 